DOI: $10.7242 / 1998-2097 / 2018.3 .2$

УДК 531 / 534: [57 + 61]

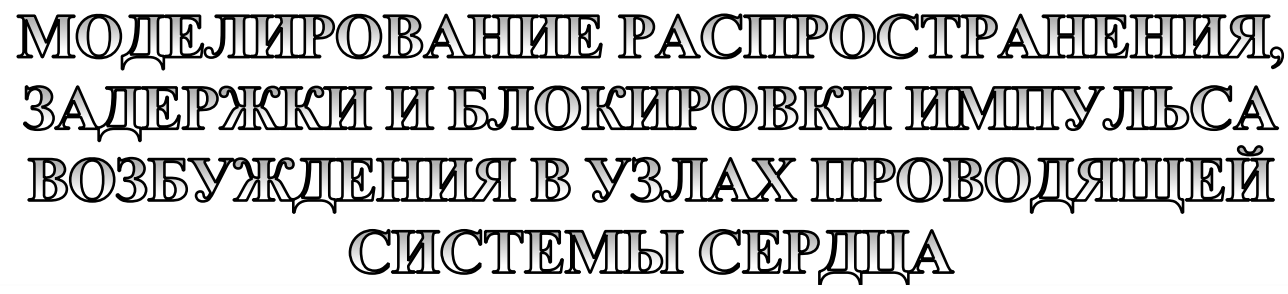

И.Н. Вассерман, Институт механики сплошных сред УрО РАН

А.П. Шестаков, Институт механики сплошных сред УрО РАН

В статье численно моделировалось распространение возбуждения в проводящей системе. Дан обзор моделей поведения трансмембранного потенциала клеток проводящей системы. Для работы были выбраны соотношения Ли-Руди (2011). Построена конечно-элементная модель проводящей системы, где она рассматривалась как одномерная последовательность клеток, соединенных на концах. На основе разработанной модели исследовался один из механизмов блокады ножек пучка Гиса. Рассмотрена Ү-образная структура, моделирующая переход от общего ствола пучка Гиса к ножкам Гиса. Результаты показывают, что в зависимости от проводимости частей проводящей системы могут иметь место три режима: прохождение возбуждения через разветвление, полная блокада и прохождение возбуждения с задержкой. Получены области значений проводимости общего ствола пучка Гиса и ножек Гиса, при которых имеет место каждый из этих режимов. Рассмотрена роль быстрых и медленных натриевых каналов в возникновении режима с задержкой.

Ключевые слова: проводящая система сердиа, блокада ножек пучка Гиса, электродинамика, метод конечных элементов.

Для сердечной деятельности характерна тесная взаимосвязь электрических, диффузионных, химических, деформационных и гидродинамических процессов. Среди процессов, происходящих в сердечной ткани, ведущую роль играет распространение электрического возбуждения. поскольку оно определяет и запускает все другие процессы в миокарде. Кроме того, опасные нарушения сердечного ритма являются одними из самых распространенных сердечнососудистых заболеваний и изучение причин их возникновения может способство- вать своевременному предупреждению этих опасных состояний. Физиологическое возбуждение желудочков сердца происходит посредством проводящей системы, скорость распространения импульса в которой на порядок превосходит таковую в миокарде. Электрическое взаимодействие клеток проводящей системы и желудочков происходит на эндокарде. Поэтому картина возбуждения будет существенно отличаться от того случая, когда первоначальное возбуждение происходит в результате внешнего воздействия на миокард в одной 
точке. Кроме того, в ряде патологических случаев возбуждение может переходить не только от проводящей системы к миокарду, но и в обратную сторону. Это явление является редким, но крайне опасным с точки зрения развития аритмий.

Для клеток проводящей системы характерны более высокая скорость возрастания трансмембранного потенциала при возбуждении, большая продолжительность его действия, более низко расположенное плато, более ярко выраженный «зуб», более выраженная зависимость длительности действия трансмембранного потенциала от частоты возбуждения, возможность низкочастотного самовозбуждения при отсутствии внешнего сигнала [1].

Поведение трасмембранного потенциала клеток проводящей системы было описано одним из первых среди всех клеток сердца в 1962 году в работе Noble [2]. Эта модель является модификацией модели Ходжкина-Хаксли [3] и также содержит всего четыре переменные: трансмембранный потенциал и три воротные переменные. Модель неплохо описывает изменение во времени, но в ней отсутствует детальное описание индивидуальных ионных токов. В [4] было сделано усовершенствование данной модели, позволяющее более точно учесть вклад индивидуальных токов и воротных переменных. При этом форма изменения трансмембранного потенциала - та же что и в [2].

В 1985 г. DiFrancesco и Noble разработали модель [5], учитывающую более тонкие механизмы, такие, как обмен ионов, ионные насосы, изменение концентрации ионов. Долгое время эта модель являлась своего рода стандартом при расчете проводящей системы сердца. Обзор ранних моделей электрофизиологии клеток проводящей системы дан в [1], там же предложено модифицировать используемые при описании желудочков модели $[6,7]$ для описания проводящей системы. Интерес к созданию моделей клеток проводящей системы возобновился только начиная с 2009 года [8-13].
Программную реализацию многих из вышеупомянутых моделей можно найти в [15] или сгенерировать из [16] с помощью Myokit [17,18].

Одной из распространенных патологий проводящей системы является блокада ножек пучка Гиса. В этом случае та область желудочка, которая должна была возбуждаться блокируемой частью проводящей системы, возбуждается через окружающие области желудочка. Это приводит к задержке возбуждения желудочка. Блокада может возникать как вследствие повреждения тканей проводящей системы, так и в ткани без видимых повреждений [1]. В вышеупомянутой работе это повреждение моделируется изменением потенциала покоя на определенном участке проводящей системы.

Здесь будет рассмотрен другой механизм возникновения блокады.

В [19] показано, что блокада может возникать при резком изменении суммарного периметра и площади сечения ветвей проводящей системы после разветвления. Кроме того, скорость распространения импульса возбуждения в общем стволе пучка Гиса в 2-4 раза ниже чем в ножках Гиса и волокнах Пуркинье [20,21].

Моделируя распространение волны возбуждения в одномерном однородном волокне без разветвлений, мы получили оценки значений проводимости, соответствующих возможным скоростям. Проводящая система здесь рассматривается как одномерная последовательность клеток, соединенных на концах. Если не учитывать градиент внеклеточного пространства, то уравнение, описывающее распространение трансмембранного потенциала имеет вид [19].

$$
p\left(C_{m} \frac{\partial V}{\partial t}+I_{i o n}\right)=\frac{\partial}{\partial x}\left(A_{p} \sigma_{p} \frac{\partial V}{\partial x}\right),
$$

где $p$ - периметр сечения, $A_{p}$ - площадь сечения клетки, $\sigma_{p}$ - усредненная проводимость волокна проводящей системы, $C_{m}$ - емкость мембраны. 
Если проводимость и сечение постоянны, то

$$
\begin{gathered}
\frac{1}{p} \cdot \frac{\partial}{\partial x}\left(A_{p} \sigma_{p} \frac{\partial V}{\partial x}\right)= \\
=\frac{A_{p}}{p} \sigma_{p} \frac{\partial^{2} V}{\partial x^{2}}=\frac{1}{\chi_{p}} \sigma_{p} \frac{\partial^{2} V}{\partial x^{2}},
\end{gathered}
$$

и (1) может быть записано в виде

$$
\chi_{p}\left(C_{m} \frac{\partial V}{\partial t}+I_{i o n}\right)=\sigma_{p} \frac{\partial^{2} V}{\partial x^{2}} .
$$

Однако в общем случае соотношение (2) не выполняется и уравнение (3) не может быть использовано. Соотношение между трансмембранным потенциалом и трансмембранным током описывалось соотношениями Ли-Руди [12]. При этом считалось, что разные части проводящей системы состоят из одинаковых клеток, а следовательно, параметры этих соотношений идентичны. Вариация скорости распространения происходила только за счет изменения проводимости (рис. 1).

Для моделирования блокады ножек пучка Гиса была рассмотрена $Y$-образная структура (рис. 2). Первоначальное возбуждение производилось в окрестности точки $A$. Так же, как и в прошлом случае, использовалась модель Ли-Руди, варьировались проводимости общего ствола и ножек пучка Гиса.

В зависимости от геометрических и физических параметров частей проводящей системы имеют место три режима: возбуждение проходит из общего ствола пучка Гиса в ножки Гиса через разветвле-

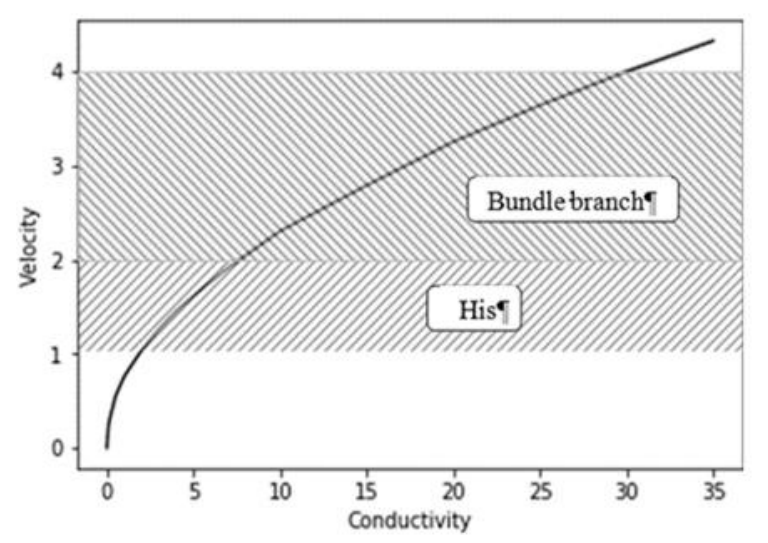

Рис. 1. Скорость распространения возбуждения и проводимость ние; возбуждение не проходит; возбуждение проходит, но с задержкой (рис. 3).

Рассмотрены области значений проводимости общего ствола пучка Гиса $\sigma_{p_{1}}$ и ножек Гиса $\sigma_{p_{2}}$, при которых имеет место каждый из этих режимов. Они показаны на рис. 4 для случая, когда диаметры корневого пучка Гиса и ножек Гиса равны. Следует заметить, что если переход от режима с задержкой к режиму полной блокады происходит резко, то переход между режимами с задержкой и без задержки является плавным. Близко к границе смены режимов задержка имеет место, но очень мала. Если диаметр ножек Гиса равен 0,7 диаметра корневого пучка Гиса, то возбуждение при отсутствии блокады быстрых натриевых каналов распространяется без задержки при всех допустимых значениях $\sigma_{p_{1}}$ и $\sigma_{p_{2}}$. При частичной блокаде быстрых натриевых каналов области соответствующих режимов изменяются (рис. 5).

Рассмотрим источник возникновения второго режима (с задержкой). Для этого в сечениях, находящихся рядом с разветвлением (рис. 6), были выведены значения трансмембранного потенциала и некоторых индивидуальных трансмембранных токов.

Ток, приводящий к режиму с задержкой, характеризуется медленной деполяризацией до значений трансмембранного потенциала, обеспечивающего быструю деполяризацию. Анализ индивидуальных токов показывает, что таким свойством обладает медленный натриевый ток.

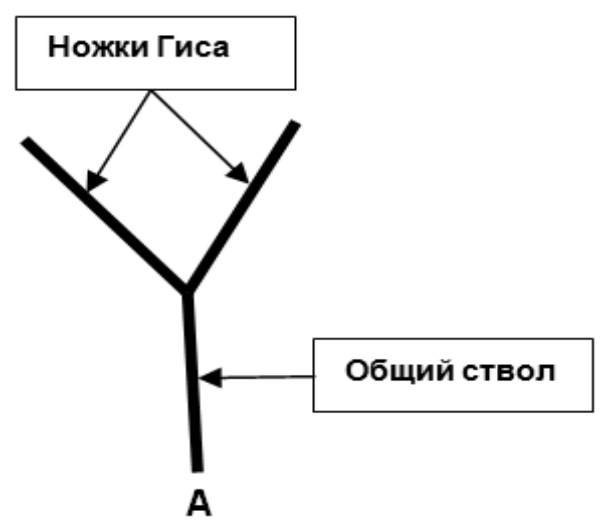

Рис. 2. Разветвление проводящей системь 


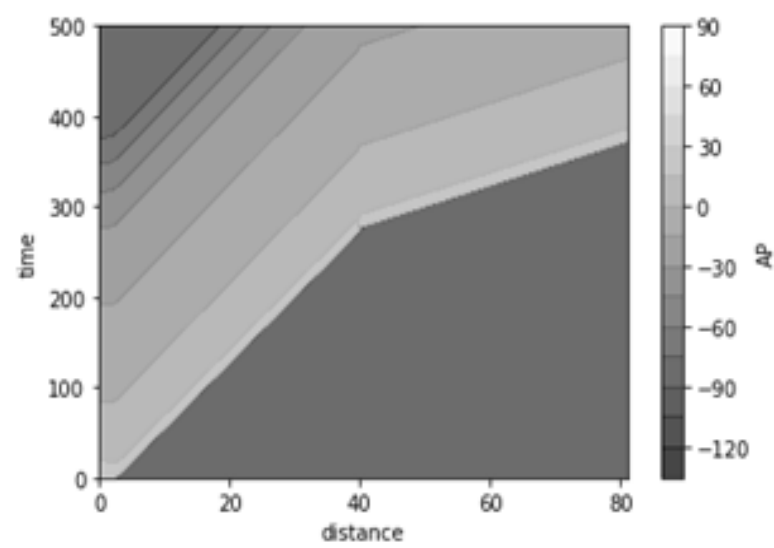

$a$

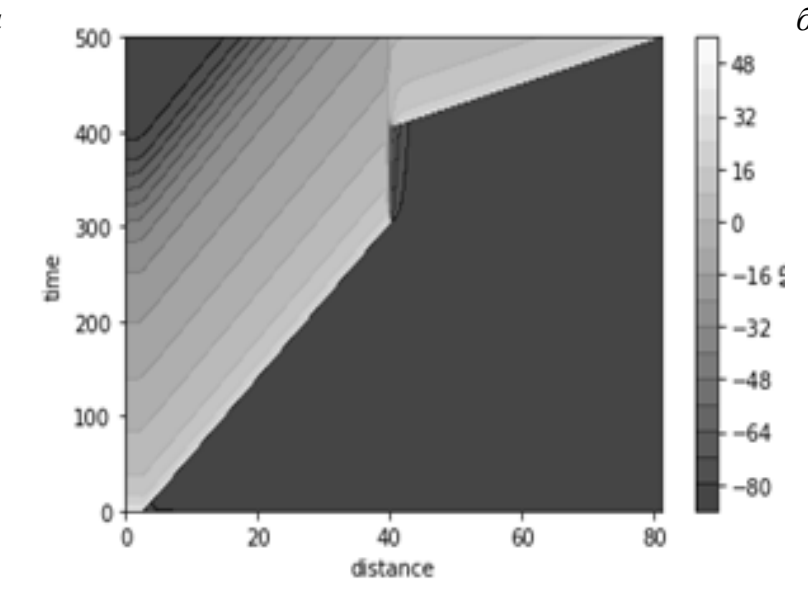

B

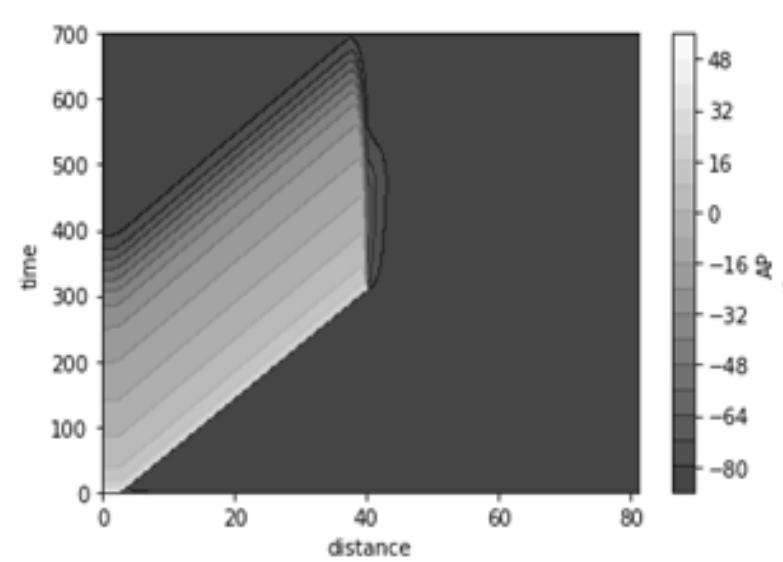

$\sigma$

Рис. 3. Прохождение возбуждения через разветвление:

a- проходит, б-не проходит, в-проходит, но с задержкой

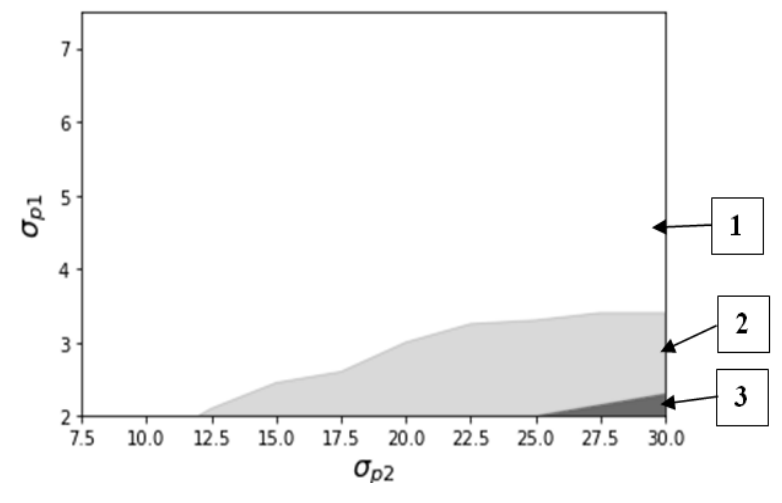

Рис. 4. Области значений проводимости общего ствола $\sigma_{p_{1}}$ и ножек пучка Гиса $\sigma_{p_{2}}$ при которых возбуждение: 1 - проходит через разветвление, 2 - проходит с задержкой, 3 - не проходит

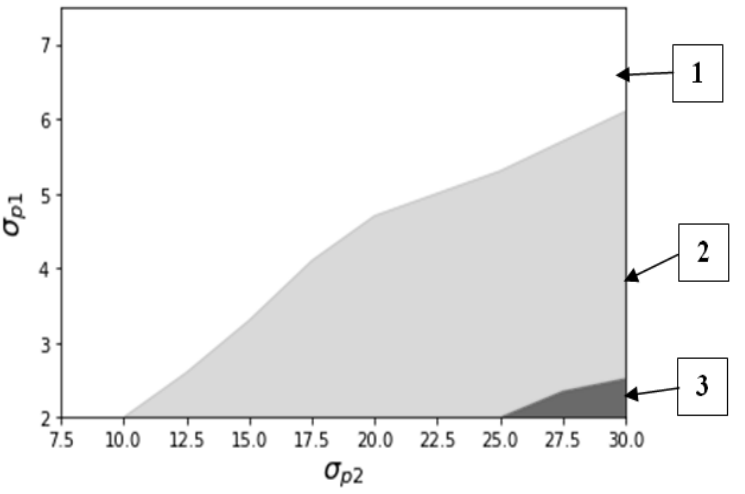

Рис. 5. Частичная блокада быстрых натриевых каналов $I_{N a} \cdot 0,6$. Области значений проводимости общего ствола пучка Гиса $\sigma_{p_{t}}$ и ножек Гиса $\sigma_{p_{2}}$ при которых возбуждение: 1 - проходит через разветвление,

2 - проходит с задержкой, 3 - не проходит 


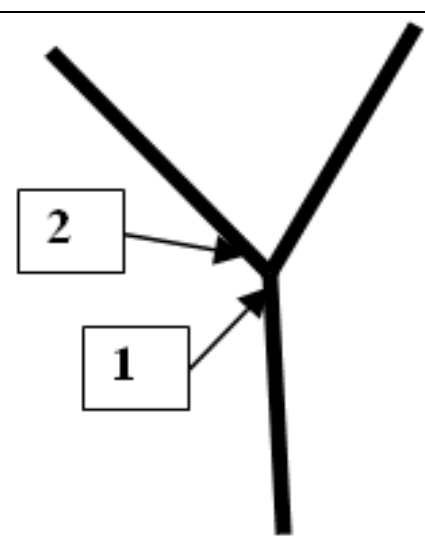

Рис. 6. Рассматриваемые сечения

Режим с задержкой возникает, когда трансмембранного потенциала, возникающего в ножках Гиса под действием электрического поля от общего ствола Гиса, недостаточно для возбуждения быстрого натриевого тока, но достаточно для возбуждения медленного натриевого тока. Последний приводит к медленной

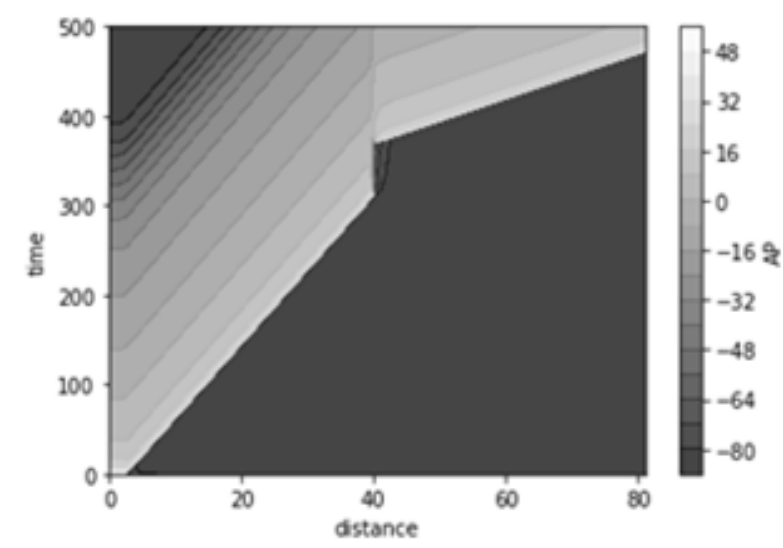

$a$
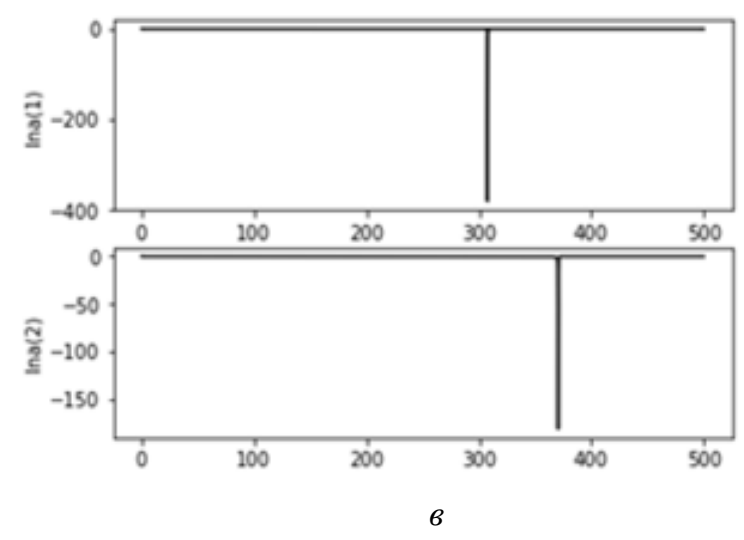

деполяризации до значений трансмембранного потенциала, достаточного для возбуждения быстрого натриевого тока, который и обеспечивает быструю деполяризацию (рис. 7).

Роль медленного натриевого тока в данном случае подтверждается еще и тем, что при изменении проводимости соответствующих каналов изменяется и продолжительность задержки (рис. 8). При этом возможен переход в другой режим, когда возбуждение проходит разветвление без задержки, или не проходит вообще (рис. 9).

Согласно [12], медленный натриевый ток состоит из двух компонент: $\mathrm{I}_{\mathrm{NaL2}}$ и $\mathrm{I}_{\mathrm{NaL3}}$. Результаты моделирования показывают, что именно $\mathrm{I}_{\mathrm{NaL} 3}$ обеспечивает медленную деполяризацию во время задержки (рис. 10).
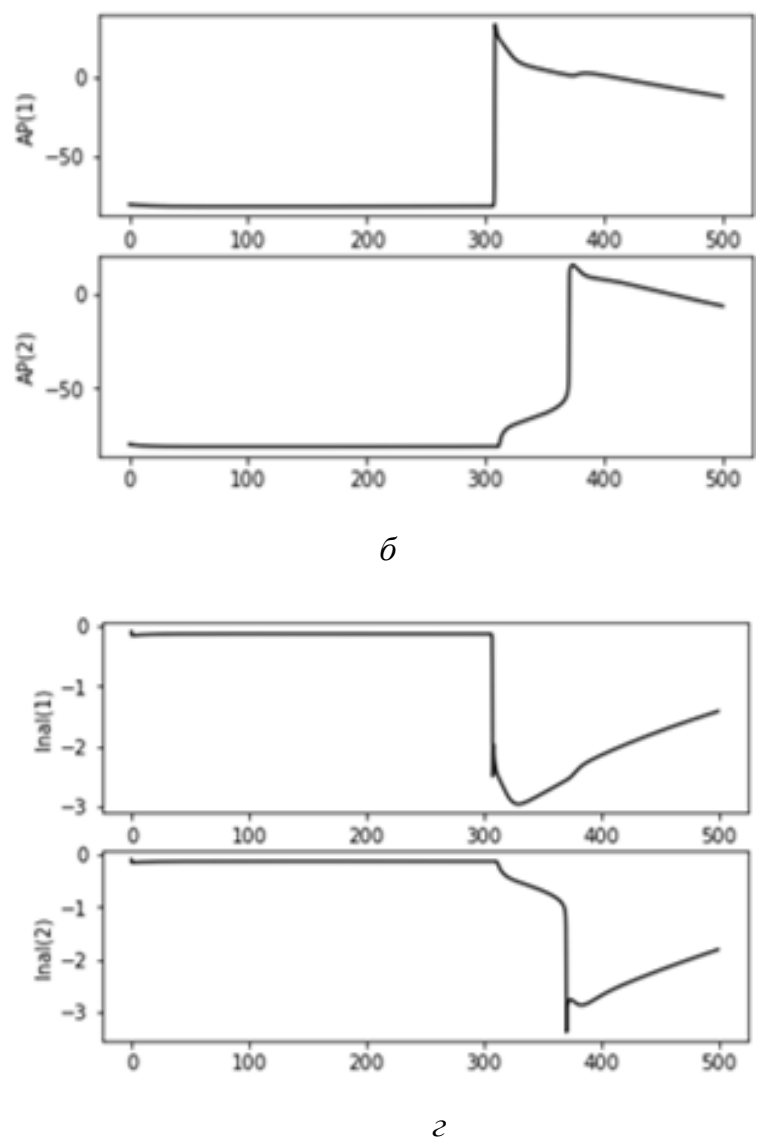

Рис. 7. Описание режима с задержкой: а-общая картина распространения; б - трансмембранный потенцииал в рассматриваемых сечениях; в - быстрый натриевый ток; г-медленный натриевый ток 


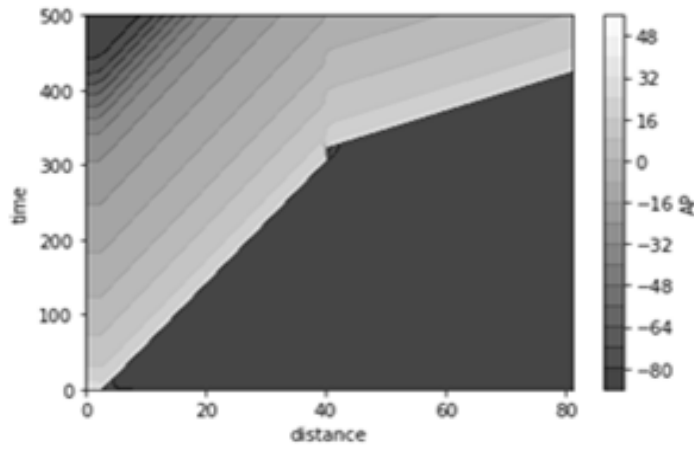

$a$
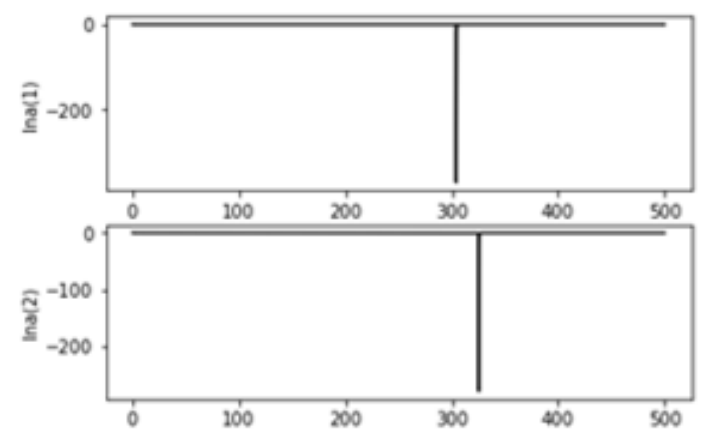

6
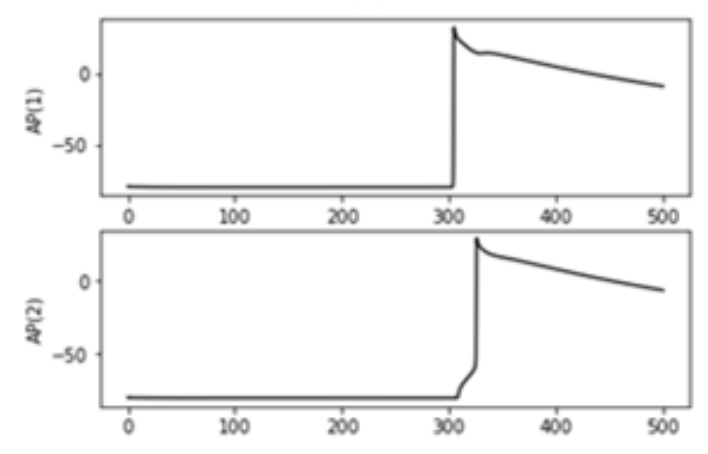

$\sigma$
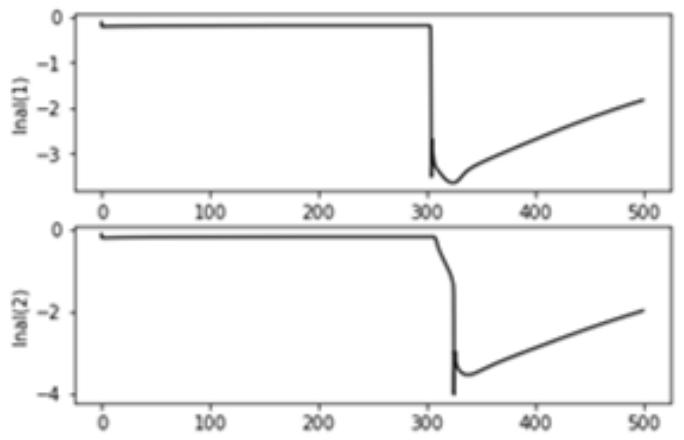

Рис. 8. Описание режима с задержкой $\left(I_{N a L} \cdot 1,4\right):$ a - общая картина распространения;

б - трансмембранный потенциал в рассматриваемых сечениях; в - быстрый натриевый ток; г-медленный натриевый ток
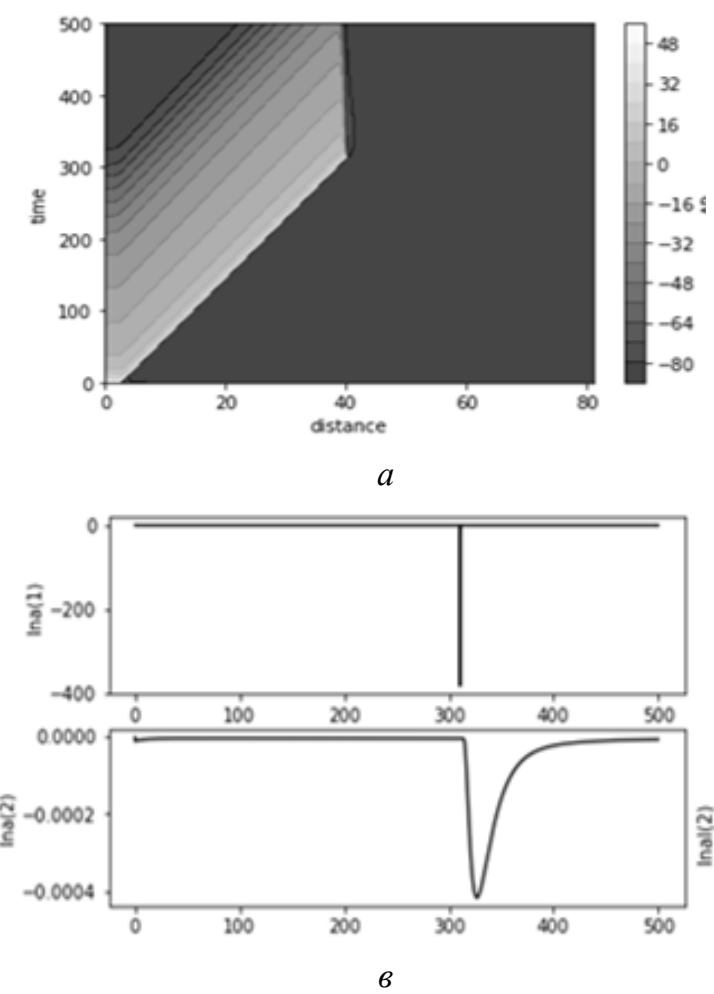
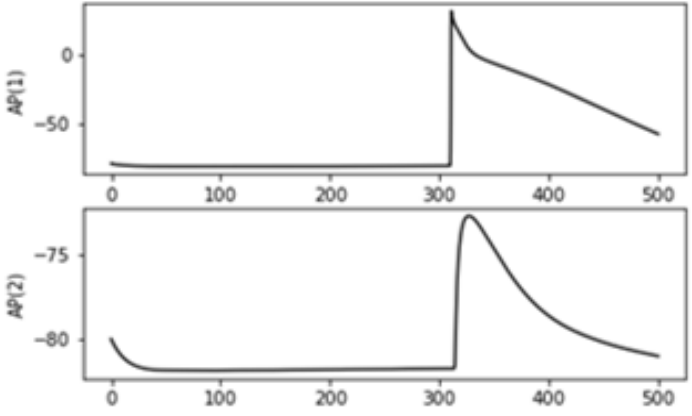

6

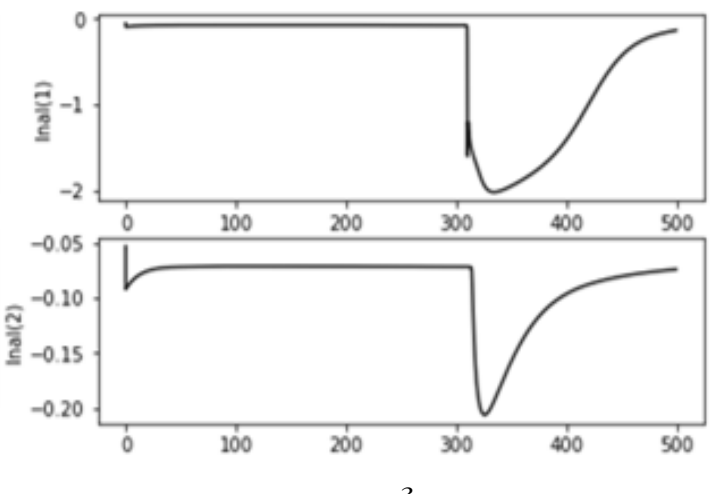

Pис. 9. Описание режима с задержкой $\left(I_{N a L} \cdot 0,6\right):$ - общая картина распространения; б - трансмембранный потенциал в рассматриваемых сечениях; в - быстрый натриевый ток; г-медленный натриевый ток 


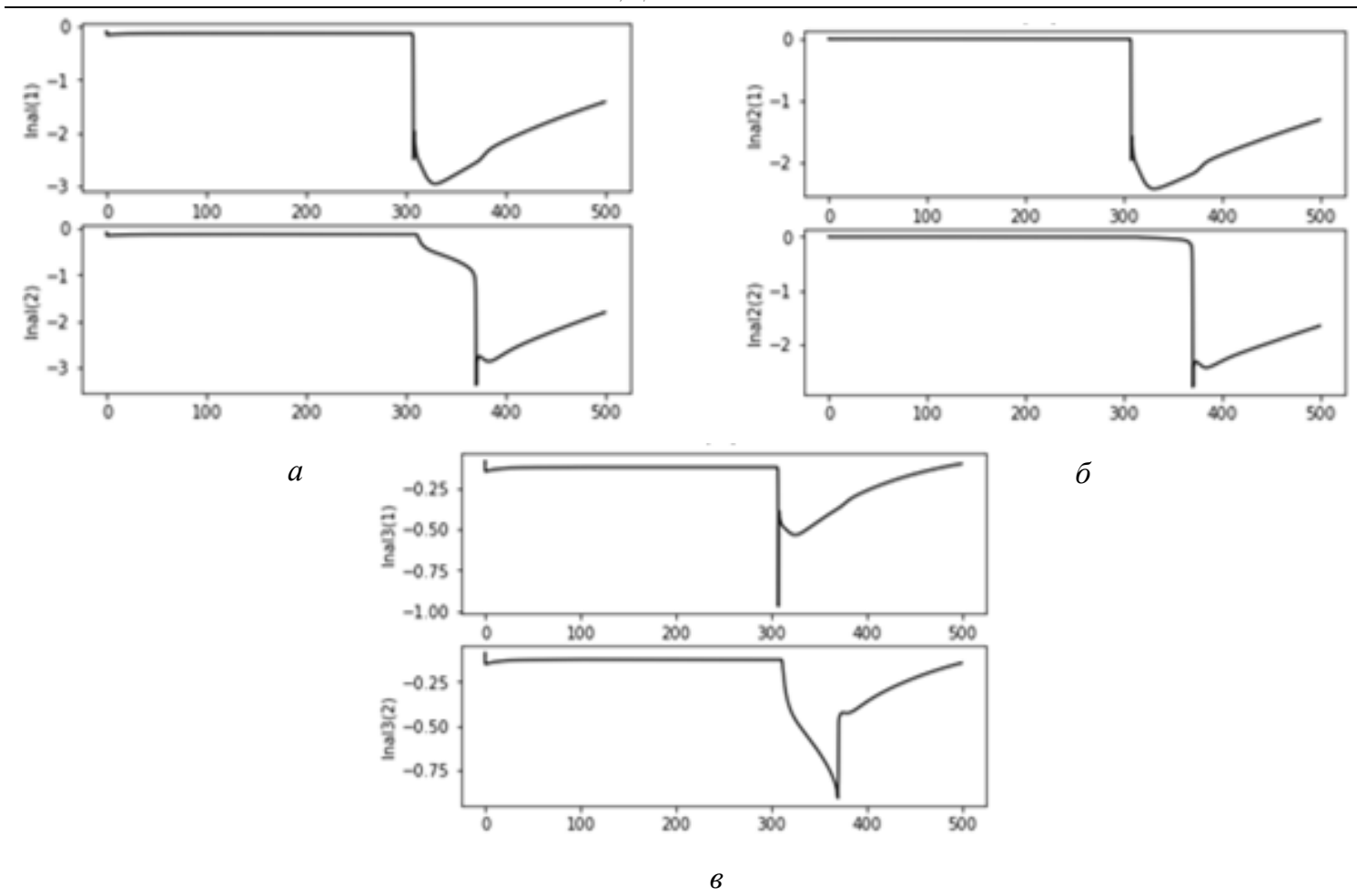

Pис. 10. Медленный натриевый ток: а - суммарный; б-компонента $I_{N a L 2}$; в - компонента $I_{N a L 3}$

\section{Библиографический список}

1. Ten Tusscher K.H.W.J., Panfilov A.W. Modelling of the ventricular conduction system // Progress in Biophysics and Molecular Biology. - 2008. - Vol. 96. - P. 152-170.

2. Noble D. A Modification of the Hodgkin-Huxley Equations Applicable to Purkinje Fibre Action and Pacemaker Potentials // J. of Physiology. - 1962. - Vol. 160. - P. 317-352.

3. Hodgkin L., Huxley A.F. A Quantitative Description of Membrane Current and its Application to Conduction and Excitation in Nerve // The J. of Physiology. - 1952. - Vol. 117. - P. 500-544.

4. McAllister R.E., Noble D., Tsien R.W. Reconstruction of the Electrical Activity of Cardiac Purkinje Fibres // J. of Physiology. - 1975. - Vol. 251. - P 1-59.

5. Francesco Di, Noble D. A Model of the Cardiac Electrical Activity Incorporating Ionic Pumps and Concentration Changes - Simulations of Ionic Currents and Concentration Changes // Phil. Trans. R. Soc. Lond. - 1985. - Vol. 307. - P. 353-398.

6. Ten Tusscher K.H.W.J., Noble D., Noble P.J., Panfilov A.V. A model for human ventricular tissue // American J. of Physiology. - 2004. - Vol. 286. - № 4. - P. 1573-1589.

7. Ten Tusscher K.H.W.J., Panfilov A.V. Alternans and spiral breakup in a human ventricular tissue model // American J. of Physiology, Heart and Circulatory Physiology. - 2006. - Vol. 291. - P. 1088-1100.

8. Aslanidi O.V., Stewart P., Boyett M.R., Zhang H. Optimal velocity and safety of discontinuous conduction through the heterogeneous Purkinje-ventricular junction // Biophysical J. - 2009. - Vol. 97. - P. 20-39.

9. Stewart P., Aslanidi O.V., Noble D., Noble P.J., Boyett M.R., Zhang H. Mathematical models of the electrical action potential of Purkinje fibre cells // Philosophical Transactions of the Royal Society. 2009. - Vol. 367. - P. 2225-2255.

10. Sampson K.J., Iyer V., Marks A.R., Kass R.S. A computational model of Purkinje fibre single cell electrophysiology: implications for the long QT syndrome // J. Physiol. - 2010. - Vol. 588. - № 14. P. 2643-2655.

11. Corrias A., Giles W., Rodriguez B. Ionic mechanisms of electrophysiological properties and repolarization abnormalities in rabbit Purkinje fibers // Am. J. Physiol. Heart Circ. Physiol. - 2011. Vol. 300. - № 5. - P. 1806-1813.

12. Li P., RudyY. A Model of Canine Purkinje Cell Electrophysiology and $\mathrm{Ca}^{2+}$ Cycling. Rate Dependence, Triggered Activity, and comparison to Ventricular Myocites // Circulation Reesearch. 2011. - Vol. 109. - P. 71-79. 
13. Cherry E.M., Fenton F.H. Contribution of the Purkinje network to wave propagation in the canine ventricle: insights from a combined electrophysiological-anatomical model // Nonlinear Dyn. - 2012. Vol. 68. - P. 365-379.

14. Garzon A., Grigoriev R.O., Fenton F.H. Continuous-time control of alternans in long Purkinje fibers // Chaos. - 2014. - Vol. 24. - № 3. - 033124.

15. URL:http://rudylab.wustl.edu/research/cell/code/AllCodes.html (дата обращения: 18.09.2018).

16. The CellML project [Электронный pecypc] URL:https://models.cellml.org/cellml (дата обращения: 18.09.2018).

17. Clerx M., Collins P., de Lange E., Volders P.G. Myokit: A simple interface to cardiac cellular electrophysiology // Progress in Biophysics and Molecular Biology. - 2016. - Vol. 120. - №1-3. - P. 100-114.

18. URL:http://myokit.org/ (дата обращения: 18.09.2018).

19. Keener J., Sneyd J. Mathematical Physiology. Springer, 2009.

20. Laske T.G., Iaizzo P.A. The Cardiac Conduction System // In: Handbook of Cardiac Anatomy, Physiology and Devices. Humana Press. - 2005. - Chapter 9. - P. 123-136.

21. Iaizzo P.A., Laske T.G. Anatomy and Physiology of the Cardiac Conduction System. // In: Cardiac Electrophysiology Methods and Models. Springer. - 2010. - Chapter 4. - P. 73-89.

\title{
MODELLING THE IMPULSE DISTRIBUTION, DELAY AND BLOCK IN THE ELECTRICAL CONDUCTION SYSTEM OF THE HEART BUNDLES
}

\author{
I.N. Vasserman, A.P. Shestakov \\ Institute of Continuous Media Mechanics UB RAS
}

In the article the excitatory irradiation in the electrical conduction system was numerically modeled. The overview of transmembrane potential of electrical conduction system was given. The Luo-Rudi model (2011) was chosen for the investigation. A finite element model of the electrical conduction system was built in which the latter was regarded as a one-dimensional sequence of cells connected at the edges. Based on the elaborated model one of the mechanisms of a bundle branch block was investigated. A Y-shaped structure which models the move from the common trunk of the bundle of His to the bundle branches was investigated. The results show that depending on the conductivity of certain parts of the electrical conduction system of the heart three regimes might occur: excitation advancing through branching, complete block or excitation advancing with delay. Conductivity Value Domains for the common trunk of the bundle of His and the bundle branches in which each of the regimes occurs were obtained. The role of quick and slow sodium channels when the regime with delay occurs is considered.

Keywords: electrical conduction system of the heart, bundle branch block, electrodynamics, finite element method.

\section{Сведения об авторах}

Вассерман Игорь Николаевич, кандидат физико-математических наук, научный сотрудник, Институт механики сплошных сред УрО РАН - филиал Пермского федерального исследовательского центра УрО РАН (ИМСС УрО РАН), 614013, г. Пермь, ул. Академика Королёва, 1; e-mail: igorw@icmm.ru

Шестаков Алексей Петрович, младший научный сотрудник, ИМСС УрО РАН; e-mail: shap@icmm.ru 